Send your research letters to the editor, British Dental Journal, 64 Wimpole Street, London W1G 8YS or by email to bdj@bda.org Priority will be given to letters less than 500 words long. Letters should be typed. Authors must sign the letter, which may be edited for reasons of space

\section{Phantom bite revisited}

Sir, just occasionally I read an article that is 'ground breaking'. I am, indeed, both grateful to and appreciative of Messrs. Jagger and Korszun (BDJ 2004,197: 241) for their paper. Certainly it does describe that nightmare scenario of continuing patient treatment with deteriorating appreciation. Both in terms of professional satisfaction and cost implications. A very worrying problem!

All three patients had symptoms suggestive of 'temporo-mandibular joint pain dysfunction' as such is mechanically caused by a tooth clenching habit. One of the products is fatigue in masticatory muscles and such results in failure of proprioceptive feedback.

Any patient who has a tooth clenching habit will admit that, on occasions, the mouth is 'uncomfortable'. Treatment modalities must therefore be directed towards the cause of such a habit, rather than merely treating the symptoms.

In this day and age we are looking at treatments, including temporomandibular joint surgery, occlusal splints and occlusal grinding. Not to mention managing traumatic pulpitis and periodontitis.

I wonder whether we could take a big leap forward, in refocusing on cause, rather than symptoms i.e. lack of proprioceptive feedback. Once we have awareness of the phenomenon, then I do believe that a great deal of investigative dental treatment would become unavoidable and 'phantom bite' explained. B. Littler

Essex

doi: 10.1038/sj.bdj.4812056

\section{Blood borne viruses}

Sir, I read with interest the paper by Professor Crossley, (BDJ 2004, 196:749) and would like to confirm that the problem of people with HIV and other blood borne viruses being refused treatment is not confined to South Cheshire. Both as a Senior Community Dentist, and as an Associate Specialist for Sheffield Teaching Hospitals, I receive a regular stream of requests to see patients where the only or main criterion is the patient's positive status for blood-borne viruses. I do, however, have to comment on the opening paragraph where the author states 'dental associations have issued recommendations indicating that dentists are obliged to provide care for patients with infectious diseases'. This is an understatement.

To quote from the GDC Maintaining Standards 'It is unethical for a dentist to refuse to treat a patient solely on the grounds that the person has a blood borne virus or any other transmissible disease or infection'1 Also, 'Failure to employ adequate methods of cross-infection control would almost certainly render a dentist liable to a charge of serious professional misconduct.' ${ }^{1}$ and 'Detailed guidance on cross-infection control has been issued ... by the BDA, and is endorsed by the council' 1

From the BDA, there is 'The medical history and examination may not identify asymptomatic carriers of infection disease and universal precautions must be adopted. This means that the same infection control procedures must be used for all patients." ${ }^{2}$

I submit that these are not recommendations but direct instructions, with the threat of sanctions against dentists who break them. There are also issues to be considered with regard to the Disability Discrimination Act.

For those dentists who cite increased cost of cross-infection control measures for these patients, my answer is straightforward - either you are simply panicking because on this occasion you know the patient's status and are going over the top, or your current measures are inadequate to meet the GDC requirements. I was most disappointed that these themes were not brought out in the paper.

I would hope that the paper and this response will enable some dentists to reconsider their position, and provide care for patients with blood borne viruses -which they are already doing, they just do not know on whom!

\section{K. I. Wilson \\ Chesterfield}

1. GDC Maintaining Standards Nov 1997, updated May 2001

2. British Dental Association Infection Control in Dentistry Feb 2003 A 12

doi: 10.1038/sj.bdj.4812057

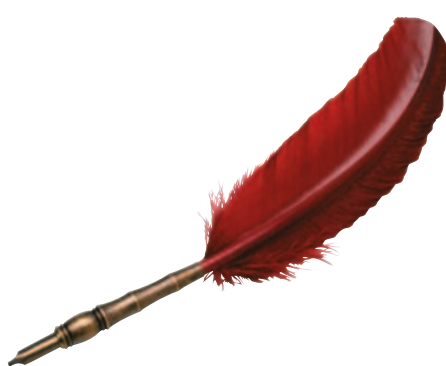

\section{Referrals}

Sir, I read with interest the letter by N J Milner and N A Smithson (BDJ 2004, 197: 372) for bringing to light the continuing problem of inappropriate referrals of wisdom teeth to oral and maxillofacial departments. Removal of wisdom teeth is one of the most common operations carried out in the U.K.; hence incorrect referrals have an impact on waiting times.

I also carried out an audit of inappropriate wisdom teeth referrals, based on the NICE guidelines, whilst working in the Oral and Maxillofacial department at Pinderfields Hospital Wakefield, in 2002. At that time, there was already a local referral protocol (predating NICE) and a referral proforma circulated to local general dental practitioners, to use when referring such patients. We decided to evaluate the impact of this protocol following the recommendations by NICE.

Consequently a prospective audit over a two month period, following Royal College of Surgeons guidelines, was carried out. This included 59 patients. In our study, the majority of referrals were found to be appropriate.

However, despite the advice, 63\% of referring practitioners did not use the proforma, instead opting for written referral letters. Interestingly, the number of referrals that were inappropriate was $6.8 \%$ and in all these cases the proforma was not used.

Such an audit not only highlighted the benefit of setting guidelines for referring practitioners to follow, but also the benefit of using tools, like a proforma, that will assist in reducing the number of inappropriate referrals.

Perhaps a standardised system of referral, using a proforma (with the current guidelines printed on it), may be helpful in reducing the wastage of NHS resources in this area.

\section{P.S. Bhogal \\ Birmingham}

1. National Institute for Clinical Excellence. Guidance on the removal of wisdom Teeth. May 2000.

doi: 10.1038/sj.bdj.4812058 